\title{
Unexplored regions of WIMP
}

\author{
Shigeki Matsumoto ${ }^{1}$ \\ Kavli IPMU (WPI), UTIAS, University of Tokyo \\ Kashiwa-no-ha 5-1-5, Kashiwa, 277-8583, Chiba, Japan \\ E-mail: shigeki.matsumoto@ipmu.jp
}

Serious searches for the weakly interacting massive particle (WIMP) have now begun. In this context, the most important questions that need to be addressed are: To what extent can we constrain the WIMP models in near future? and What will then be the remaining unexplored region in the WIMP parameter space for each model? In our quest to answer these questions, we classify WIMP in terms of quantum number and study each case adopting minimality as a guiding principle. We first study one of the simple cases of the minimal composition in the well-tempered fermionic WIMP regime, namely the singlet-doublets WIMP model. Next, we consider the nonwell-tempered fermionic WIMP regime where the WIMP is almost described by a SU(2)L gauge eigenstate. We first study the WIMP whose weakcharge is almost one as the minimal case, namely the $\mathrm{SU}(2)_{\mathrm{L}}$-triplet WIMP model. After that, we discuss the other models where WIMP carries no weakcharge, namely (almost) the standard model (SM) singlet WIMP.

In the above models, we consider all available constraints from direct and indirect searches and also the predicted constraints coming from near future. We thus obtain the present status and near future prospect of the models in all their generalities. We find that in the near future, the fermionic singlet-doublets WIMP model will be constrained almost solely by near future direct dark matter detection experiments (as compared to the weaker indirect and collider constraints) and the cosmological (relic density) constraints, and will hence be gradually pushed to the corner of the coannihilation region, if no WIMP signal is detected. Future lepton colliders will then be useful in exploring this region not constrained by any other experiments. On the other hand, most of the parameter region of the fermionic SU(2)L-triplet WIMP model will not be efficiently covered by near future collider and direct detection experiments. The remaining parameter region should then be covered by indirect detection experiments utilizing the gamma-ray observation of dwarf spheroidal galaxies (dSphs) by understanding the dark matter distribution in each dSphs very well. Concerning the SM singlet WIMP models, it turns out that wide parameter regions of the models will be covered by near future experiments. Leptophilic WIMP regime will then remain unexplored if no WIMP signal is detected, and future lepton colliders will be mandatory to explore the remaining region, as in the case of the singet-doublets WIMP model.

The 3rd International Symposium on "Quest for the Origin of Particles and the Universe"

5-7 January 2017

Nagoya University, Japan

\section{${ }^{1}$ Speaker}




\section{Introduction}

There is no doubt about the existence of dark matter in our universe thanks to astrophysical and cosmological observations ranging from galactic to cosmological scales. The nature of dark matter, however, still remains as one of the biggest mysteries of particle physics in spite of tremendous efforts to ascertain its nature since several decades. What we currently know is that the dark matter in our universe has an abundance of $\Omega_{\mathrm{c}} \mathrm{h}^{2}=0.1198$ (15) at present and that it interacts gravitationally. The dark matter is expected to be some new particle which is electrically neutral and colorless. Among several candidates, the Weakly Interacting Massive Particle (WIMP) is one of the most attractive candidates because of its simplicity and predictability. The thermal freeze-out mechanism of WIMP, which is nowadays called the WIMP miracle, naturally explains the abundance observed today when its mass is around the electroweak scale. This may open a way to understand the origin of the electroweak symmetry breaking (EWSB). Moreover, we are hopeful to detect the WIMP, because some of the predicted interactions between the WIMP and the standard model (SM) particles provide a strong driving force of WIMP detections at highenergy lepton and hadron colliders, underground laboratories (direct dark matter detections) and in cosmological/astrophysical observations (indirect dark matter detections).

We have, on the other hand, not obtained any conclusive signatures of the WIMP so far, and it pushes the celebrated WIMP scenarios gradually to the corner. This fact, however, also means that the era of serious WIMP searches has begun, so that currently the most important questions are: To what extent can we constrain the WIMP models in near future? and What will then be the remaining unexplored regions in the WIMP parameter space for each of these models? If the simplicity and predictability of the WIMP are getting lost, such theories will become less attractive. If it is not, we should consider what kinds of experiments are required to cover the unconstrained parameter region. Several big experiments, such as multi-tons scale direct dark matter detections [1, 2] and future lepton colliders [3, 4, 5], are being proposed, and it is worth figuring out what roles these experiments can play in this direction. The goal of this paper is to try and answer certain aspects of the questions by performing a comprehensive analysis.

We take a standard strategy to study such models based on the WIMP's quantum number. Once the spin of the WIMP is fixed, the WIMP field can always be written as a linear combination of colorless representations of the SM gauge group involving the electrically neutral components:

$$
\operatorname{WIMP}(\mathrm{x})=\sum_{i} z_{i}\left[\chi_{i}(x)\right]_{N . C .}
$$

with the subscript 'N.C.' indicating an electrically neutral component of the representation $\chi_{i}(x)$. The coefficient $z_{\mathrm{i}}$ follows the sum rule $\sum_{i}\left|z_{i}\right|^{2}=1$ because of the canonical-normalization of the field, and $\left|z_{i}\right|^{2}$ measures how much the representation $\chi_{i}(x)$ is involved as a component of the WIMP field. The most straightforward way for studying such WIMP scenarios is to write down an effective Lagrangian of the WIMP field involving all interactions which can be responsible for the WIMP miracle phenomenon, though it is not practical because we must introduce infinitely many representations and interactions. Instead, we try to cover the theory space by an infinite number of small patches based on the quantum number of WIMP, and construct an appropriate effective Lagrangian in each patch. However, there are still infinitely many possibilities of the 
effective Lagrangian in each patch; we can always construct the Lagrangian with any desired complexity. We therefore take simplicity as a guiding principle to write down the Lagrangian.

The WIMP models can be divided into two categories: one in which the WIMP is described almost by a gauge eigenstate of the $\mathrm{SU}(2)_{\mathrm{L}}$ interaction, namely one of the $\left|\mathrm{Z}_{\mathrm{i}}\right|^{2} \mathrm{~S}$ is close to unity. The other is the one in which the WIMP is not described by a single SU(2)L gauge eigenstate. It indicates that none of the $\left|\mathrm{Z}_{\mathrm{i}}\right|^{2} \mathrm{~S}$ are close to unity. This is nowadays referred to as the well-tempered WIMP. The former category is further divided into many patches: when a $\mathrm{SU}(2)_{\mathrm{L}}$ singlet component dominates, the so called singlet WIMP is realized. The bino-like neutralino in the minimal supersymmetric standard model (MSSM) and the Kaluza-Klein photon in the universal extra-dimension model (UED) are good examples. When the $\mathrm{SU}(2)_{\mathrm{L}}$ doublet components dominate, the doublet WIMP is obtained. Examples of such a WIMP are the Higgsino-like neutralino in the MSSM and the inert Higgs doublet in some two Higgs doublet models (2HDMs). We have the triplet WIMP when a SU(2)L triplet component dominates, with a famous example being the wino-like neutralino in the MSSM. A WIMP which is described by a higher multiplet is nowadays called the minimal dark matter. WIMP in the latter category, namely the well-tempered WIMP, is more complicated to be classified. A systematic method to decompose it into patches can be based on the number of representations that are participating in for describing the welltempered WIMP. For example, it follows $\left|\mathrm{z}_{\mathrm{i}}\right|^{2}+\left|\mathrm{z}_{\mathrm{j}}\right|^{2} \sim 1$ when this number is two with both $\left|\mathrm{z}_{\mathrm{i}}\right|^{2}$ and $\left|z_{j}\right|^{2}$ being not close to unity, $\left|z_{i}\right|^{2}+\left|z_{j}\right|^{2}+\left|z_{k}\right|^{2} \sim 1$ when the number is three, and so on. The first example, say the minimal composition here, is usually adequate to capture the typical nature of the well-tempered WIMP. Moreover, it is indeed realized in most of the parameter regions of realistic new physics models, even though we should not deny interesting possibilities that WIMPs described by many representations can potentially show some new features that are not seen in the minimal composition. Here, it is worth pointing out how coannihilation regions are incorporated in this framework. This framework automatically involves coannihilations between components inside a SU(2) $\mathrm{L}$ multiplet and between particles which are mixed with each other in the well-tempered WIMP. On the other hand, other coannihilations, such as the one between WIMP and a particle which resides in a different $\mathrm{SU}(2)_{\mathrm{L}}$ multiplet and is not mixed with the WIMP, should be considered in each individual patch, even though such a coannihilation region usually does not appear in the minimal setup of the effective Lagrangian in the patch.

The above classification of WIMP can be visualized by the figure below, where the numbers $\mathrm{X}$ and $\mathrm{Y}$ in the form of $\mathrm{X}_{\mathrm{Y}}$ are the $\mathrm{SU}(2)_{\mathrm{L}}$ and $\mathrm{U}(1)_{\mathrm{Y}}$ quantum numbers of WIMP, respectively. In the following sections, we consider fermionic WIMP models in the $\mathrm{SU}(2)_{\mathrm{L}}$ singlet, triplet, and singlet-doublet regimes (denoted by orange circles), as a demonstration of our WIMP study. We show the present status of the models in their general parameter spaces, how they will change in near future, and what will be the parameter spaces remaining in the future if no WIMP signal is detected by then. All the results are obtained by a numerical likelihood analysis scanning the parameter spaces of the WIMP models based on the experimental constraints from dark matter relic density, direct dark matter detections, indirect dark matter detections and those from

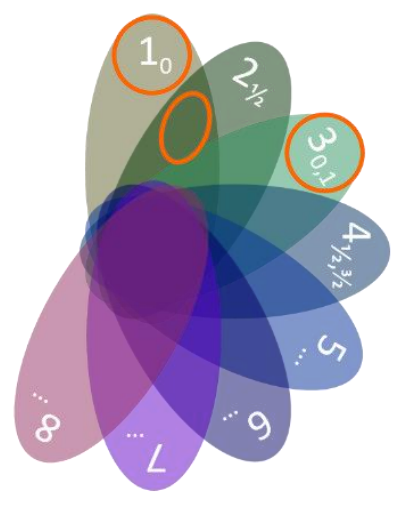
high energy colliders. We then discuss the kind of experiments and/or observations required to efficiently explore the yet unconstrained parameter regions of the models in the near future. 


\section{Fermionic singlet-doublets WIMP model}

We first discuss the singlet-doublets WIMP model, namely the simplest WIMP model in the well-tempered regime. Its minimal setup is composed of three Weyl fermions $S, D_{1}$ and $D_{2}$ with their quantum numbers $1_{0}, 2_{1 / 2}$ and $2-1 / 2$, respectively, and described by the following Lagrangian:

$$
\mathcal{L}=\mathcal{L}_{S M}+\mathcal{L}_{k i n}-\left[\frac{1}{2} M_{S} \mathrm{SS}+M_{D} \mathrm{D} \cdot \mathrm{D}+y_{1} \mathrm{~S} D_{1} \cdot H^{c}+\mathrm{y}_{2} \mathrm{SD}_{2} \cdot \mathrm{H}+\text { H. c. }\right]
$$

with $\mathcal{L}_{S M}$ and $\mathcal{L}_{\text {kin }}$ being the SM Lagrangian and the kinetic term of the three fermion fields. The Lagrangian involves all renormalizable interactions by taking the $Z_{2}$ odd property of the fermion fields into account, where the $Z_{2}$ symmetry is implicitly imposed in the above Lagrangian to guarantee the stability of the WIMP; the three new fermions are odd under the symmetry, while even for all the SM particles. The dot indicates the contraction of the $\mathrm{SU}(2)_{\mathrm{L}}$ indices via the antisymmetric tensor. The Higgs field is denoted by $\mathrm{H}$ with its quantum number $2 \frac{1}{2}$, while $\mathrm{H}^{\mathrm{c}}$ is its conjugate with the quantum number $2_{-1 / 2 .}$ See Ref. [6] for more details of the WIMP model.

We have four complex parameters $\mathrm{y}_{1}, \mathrm{y}_{2}, \mathrm{M}_{\mathrm{S}}$ and $\mathrm{M}_{\mathrm{D}}$. Three of their phases are rotated away by redefining the three Weyl fermion fields, and one phase remains as a physical one. When the phase is neither 0 nor $\pi$, the interactions violate CP symmetry. In our analysis, we take the phase to be 0 or $\pi$ for simplicity, and scan the model parameter space, assuming UV theory behind the above Lagrangian is weak-interacting, namely $\left|y_{1,2}\right|<1$. We evaluate the likelihood function, which is constructed based on various (possible) experimental data at present (in future), using the MultiNest sampling algorithm. See Ref. [6] for more details of the constraints we used.

The present status and future prospect (when no WIMP signal is detected) of the WIMP model are shown in the left and right panels of the figure below, where the scanning result is projected onto the plane of the WIMP mass $\left(\mathrm{M}_{\mathrm{N} 1}\right)$ and the doublet mass $\left(\mathrm{M}_{\mathrm{D}}\right)$, with the yellow and blue bands being $1 \sigma$ and $2 \sigma$ contours, respectively. Dark matter relic density and direct dark matter detection constraints mainly determine the shape of the survival region in both panels. Only narrow strip regions are survived in the right panel, where all the new particles including the WIMP are degenerate in mass. Future lepton colliders or bigger direct detection experiments are required to investigate this remaining parameter region. Importantly, the trend of the result (present status and future prospect) seems common for all WIMP models in the well-tempered regime, because the origin of the well-temperedness (the large mixing between different representations) is the same as that of the strong signal at direct detection experiments (the large scattering cross section between the WIMP and a nucleon), namely the Yukawa coupling $\mathrm{y}_{1,2}$.
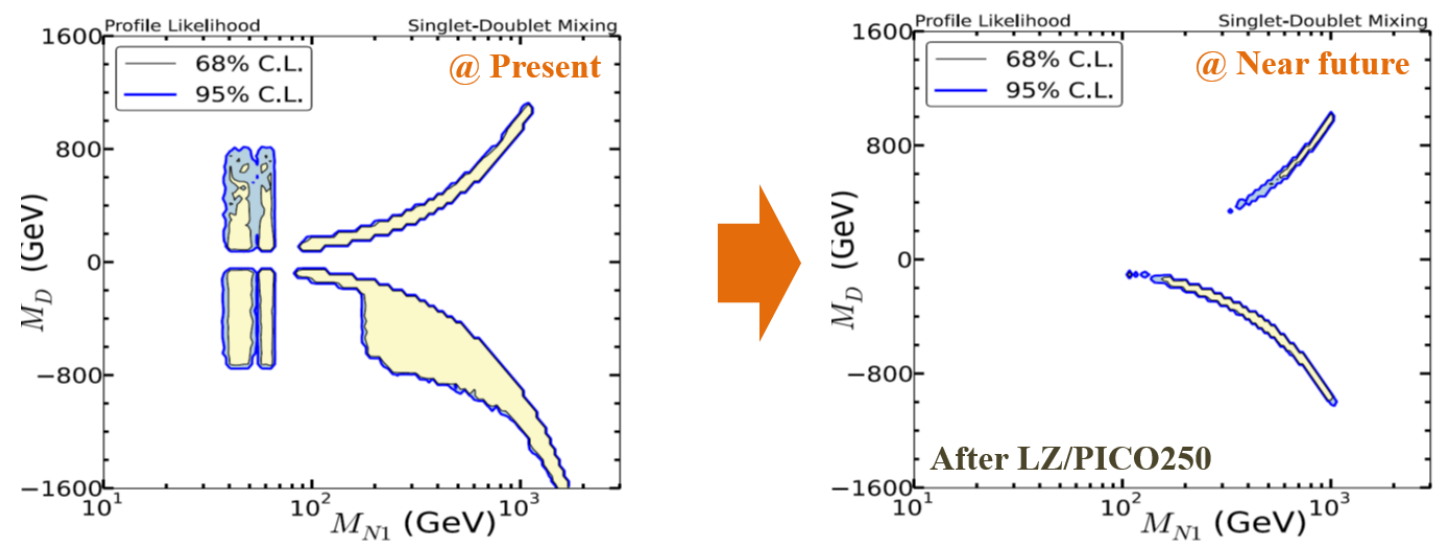


\section{Fermionic triplet WIMP model}

We next discuss the fermionic triplet WIMP model, which is known to be one of the simplest WIMP models. Its setup is, in fact, very minimal; it is enough to introduce one Weyl fermion multiplet $\mathrm{T}$ with quantum number $3_{0}$, and its dynamics are described by the following Lagrangian:

$$
\mathcal{L}=\mathcal{L}_{S M}+\bar{T}\left(i \gamma^{\mu} D_{\mu}-\mathrm{M}_{\mathrm{T}}\right) T
$$

where a $Z_{2}$ symmetry is imposed to guarantee the stability of the WIMP, as in the previous case. The neutral component of the triplet plays the role of WIMP, while other two components form an electrically charged Dirac fermion; the SU(2) L partner of the WIMP. Though it is also possible to add higher-dimensional operators to the Lagrangian to include the small mixing effect between the triplet and other $\mathrm{SU}(2)_{\mathrm{L}}$ representations, its contribution is far significant. One might think that such a contribution may affect the mass difference between the neutral and charged components, say the custodial symmetry breaking, but it is from a dimension-7 operator and quickly decoupled when the cutoff scale is larger than $\mathrm{M}_{\mathrm{T}}$. The physics of the WIMP is hence governed by the $\mathrm{SU}(2)_{L}$ gauge interaction in $\mathrm{D}_{\mu}$ and $\mathrm{M}_{\mathrm{T}}$ is the only new physics parameter.

The present status and future prospect (when no WIMP signal is detected) of the WIMP model are shown in the left and right panels of the figure below, where the result is shown on $\mathrm{M}_{\mathrm{T}}$ $\left(=\mathrm{m}_{\mathrm{X}}\right)$ axis with the corresponding self-annihilation cross section. If the relic dark matter density constraint is considered, $\mathrm{M}_{\mathrm{T}}$ is fixed to be about $3 \mathrm{TeV}$, as shown by the orange band in both panels. The LHC experiment is officially excluded the region below $270 \mathrm{GeV}$ (the dark grey region in both panels), and it will be extended to around $500 \mathrm{GeV}$ in near future if no WIMP signal is detected (the blue region in the right panel). Both are, however, much away from the $3 \mathrm{TeV}$ region. Moreover, the scattering cross section between the WIMP and a nucleon is predicted to be about $10^{-11} \mathrm{pb}$ with irrespective to $\mathrm{M}_{\mathrm{T}}$ [7], and it is again much below the current limit of direct detection experiments. On the other hand, indirect detections seem hopeful, for the self-annihilation cross section of the WIMP is boosted by the so-called Sommerfeld effect [8], as shown in the black line in both panels. Among various indirect detections, the one utilizing the gamma-ray observation of dwarf spheroidal galaxies (dSphs) is expected to give a robust opportunity to search for the WIMP (the green line in both panels), though some efforts are still needed to cover the $3 \mathrm{TeV}$ region and understanding the dark matter distribution in each $\mathrm{dSph}$ is mandatory [9]. Otherwise, more energetic collider experiment (e.g. HE-LHC or FCC-hh) or 100ton scale direct dark matter detection experiment (e.g. DARWIN) is required to cover the $3 \mathrm{TeV}$ WIMP region.
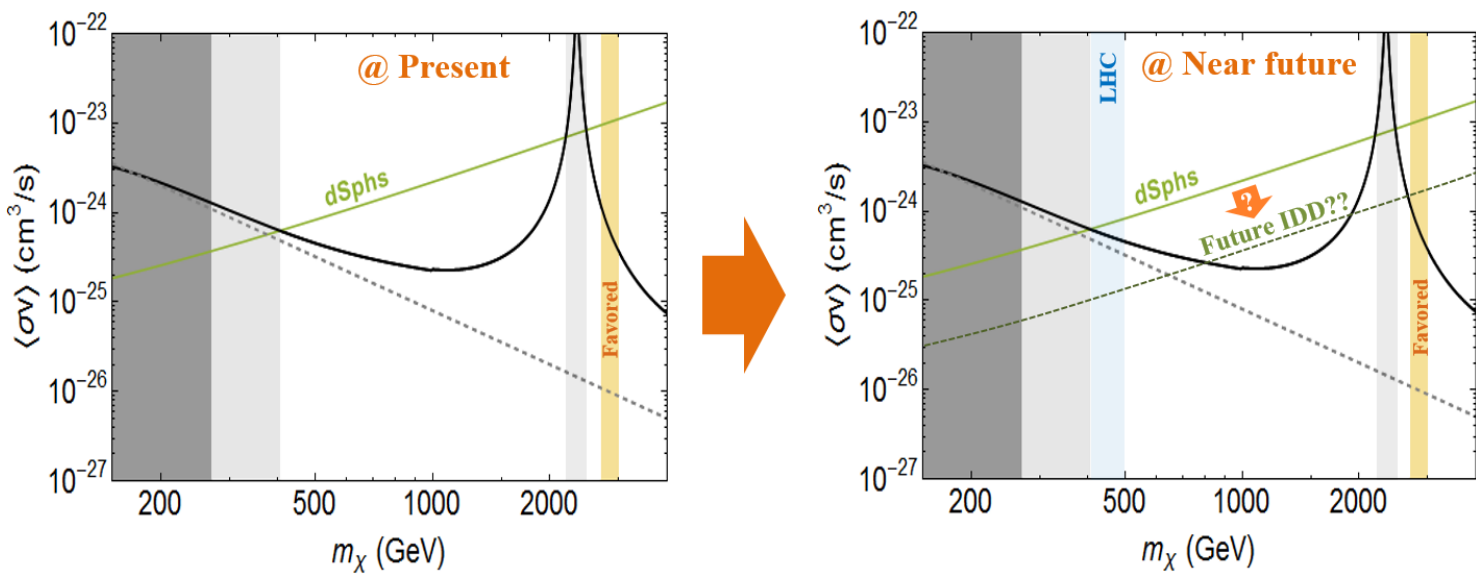


\section{Fermionic singlet WIMP models}

We finally discuss fermionic singlet WIMP models. Considering the WIMP + SM system and imposing the $\mathrm{Z}_{2}$ symmetry for the WIMP stability, no renormalizable interaction involving both WIMP and SM fields exists due to the SM gauge symmetry. It means that at least one more new particle must be introduced to have such an interaction, where this new particle is called the mediator particle. WIMP physics hence depends strongly on the property of the mediator particle, and we must consider each case of this new particle individually to discuss the singlet WIMP. Fortunately, when the mass of the mediator particle is well heavier than the WIMP and the electroweak scale, all possible cases are able to be discussed simultaneously, for the cases eventually lead to the common effective Lagrangian after integrating the mediator particle out:

$$
\mathcal{L} \supset \frac{c_{S}}{2 \Lambda}(\bar{\chi} \chi)|\mathrm{H}|^{2}+\frac{c_{P S}}{2 \Lambda}\left(\bar{\chi} i \gamma_{5} \chi\right)|\mathrm{H}|^{2}+\frac{c_{H}}{2 \Lambda}\left(\bar{\chi} \gamma^{\mu} \gamma_{5} \chi\right)\left(\mathrm{H}^{\dagger} \mathrm{i} \overleftrightarrow{D} \mathrm{H}\right)+\sum_{f} \frac{c_{f}}{2 \Lambda}\left(\bar{\chi} \gamma^{\mu} \gamma_{5} \chi\right)\left(\bar{f} \gamma_{\mu} \mathrm{f}\right)
$$

where $f$ is $Q_{i}, U_{i}, D_{i}, L_{i}$ and $E_{i}$ with those being quark doublets, up-type quark singlets, down-type quark singlets, lepton doublets and charged lepton singlets, respectively, while the subscript ' $i$ ' represents the flavor index. Coupling constant of each interaction is denoted by $c_{i}$, and $\Lambda$ is the cutoff scale of the effective Lagrangian. The model parameter space is scanned in our analysis assuming the $\mathrm{CP}$ invariance $\left(\mathrm{c}_{\mathrm{PS}}=0\right)$ and the flavor universality (flavor independent four-Fermi interactions) for simplicity. Moreover, since the use of the effective Lagrangian is not appropriate to calculate high energy collider signals, we also use several simplified models reproducing the above interactions in the decoupling limit to avoid the overestimate of the signals. See Ref. [10] for more details of the models, the prescription of the scanning and the constraints we used.

The present status and future prospect (when no WIMP signal is detected) of the WIMP models are shown in the left and right panels of the figure below, where the scanning result is projected onto the plane of the WIMP mass $\left(\mathrm{m}_{\chi}\right)$ and the cutoff scale $(\Lambda)$, with the yellow and blue bands being $1 \sigma$ and $2 \sigma$ contours, respectively. Comparison between the two panels shows that the wide parameter region will be covered by near future. The Higgs resonance region $\left(\mathrm{m}_{\chi} \sim\right.$ $\mathrm{m}_{\mathrm{h}} / 2$ ), which is survived until today as shown in the left panel, completely disappears in the right panel, meaning the region will be searched for in near future. It then turns out that the $Z$ boson resonance region $\left(\mathrm{m}_{\chi} \sim \mathrm{m}_{\mathrm{Z}} / 2\right)$ where the $\chi-\chi-Z$ coupling from the dimension- 6 interaction with the Higgs boson governs physics of the WIMPs, and the region with $\Lambda<10 \mathrm{~m}_{\chi}$ where the fourFermi interactions with leptons dominate, remain unexplored. Future lepton colliders or sensitive spin-dependent direct dark matter detection experiments are required to cover the two regions.
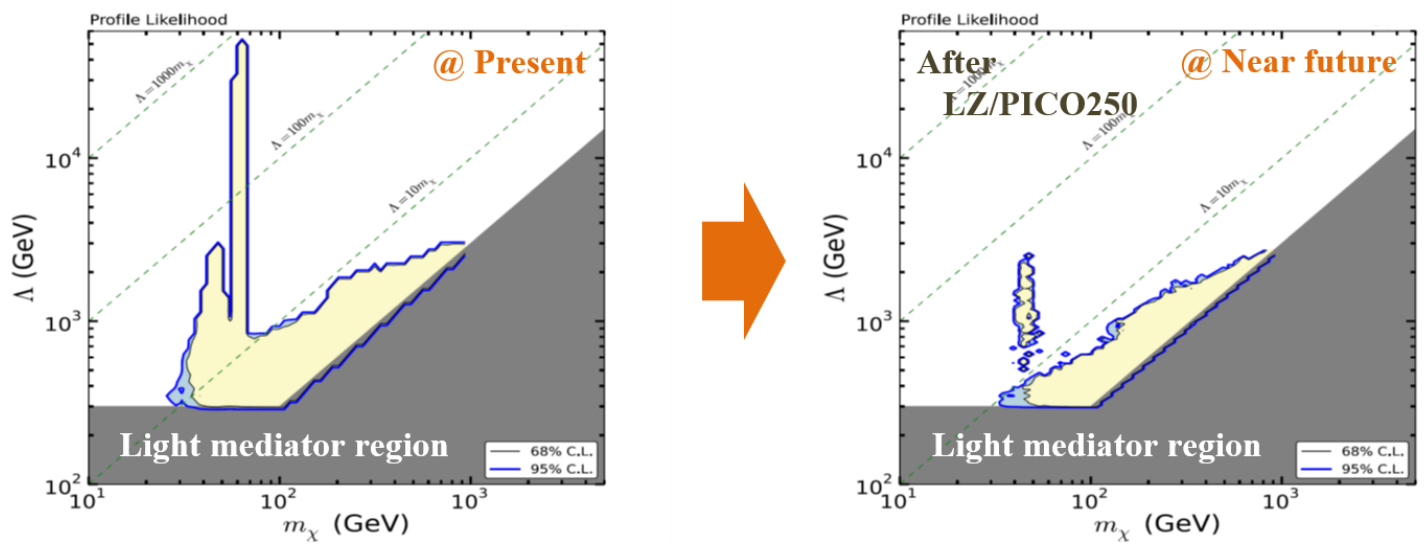


\section{Summary}

We have proposed the framework to study WIMP candidates without relying on any specific new physics models (SUSY, etc.) by classifying those based on quantum number. We particularly discuss fermionic WIMPs in the singlet-doublet, the triplet, and the singlet regimes as a demonstration of our study. The singlet-doublet WIMP is the simplest candidate in the welltempered regime. Direct detection experiments play the most important role to search for the WIMP. Thought the coannihilation region remains unexplored, it can be covered by future lepton colliders or massive direct detections. The triplet WIMP is the simplest candidate among all WIMPs. Only indirect detections, especially the gamma-ray observation of dSphs, seems to have an opportunity to hunt the WIMP thanks to the Sommerfeld effect. The singlet WIMPs require an additional new particle, the mediator, to have a renormalizable interaction with the SM particles. Though wide model parameter region will be covered by near future, future lepton colliders or massive spin-dependent direct detections are required to complete the search.

\section{References}

[1] D. C. Malling et al., After LUX: The LZ Program, [arXiv:1110.0103].

[2] DARWIN consortium, XENON Collaboration, A. Kish, Direct Dark Matter Detection with the XENON and DARWIN experiments, PoS TIPP2014 (2014) 164.

[3] T. Behnke, J. E. Brau, B. Foster, J. Fuster, M. Harrison, J. M. Paterson, M. Peskin, M. Stanitzki, N. Walker, and H. Yamamoto, The International Linear Collider Technical Design Report - Volume 1: Executive Summary, [arXiv:1306.6327].

[4] C.-S. S. Group, CEPC-SPPC Preliminary Conceptual Design Report. 1. Physics and Detector.

[5] TLEP Design Study Working Group Collaboration, M. Bicer et al., First Look at the Physics Case of TLEP, JHEP 01 (2014) 164 [arXiv:1308.6176].

[6] S. Banerjee, S. Matsumoto, K. Mukaida and Y. L. S. Tsai, WIMP Dark Matter in a Well-Tempered Regime: A case study on Singlet-Doublets Fermionic WIMP, JHEP 1611 (2016) 070 [arXiv:1603.07387].

[7] J. Hisano, K. Ishiwata, N. Nagata, QCD Effects on Direct Detection of Wino Dark Matter, JHEP 1506 (2015) 097 [arXiv:1504.00915].

[8] J. Hisano, S. Matsumoto and M. M. Nojiri, Explosive dark matter annihilation, Physical Review Letters 92, (2004) 031303 [hep-ph/0307216]; J. Hisano, S. Matsumoto, M. M. Nojiri and O. Saito, Non-perturbative effect on dark matter annihilation and gamma ray signature from galactic centre, Physical Review D71 (2005) 063528 [hep-ph/0412403].

[9] K. Hayashi, K. Ichikawa, S. Matsumoto, M. Ibe, M. N. Ishigaki and H. Sugai, Dark matter annihilation and decay from non-spherical dark halos in galactic dwarf satellites, Monthly Notices of the Royal Astronomical Society 461 (2016) 2914 [arXiv:1603.08046]; Foreground effect on the $J$-factor estimation of classical dwarf spheroidal galaxies, to be published in Monthly Notices of the Royal Astronomical Society [1608.01749].

[10] S. Matsumoto, S. Mukhopadhyay and Y. L. S. Tsai, Singlet Majorana fermion dark matter: a comprehensive analysis in effective field theory, JHEP 1410 (2014) 155 [arXiv:1407.1859]; S. Matsumoto, S. Mukhopadhyay and Y. L. S. Tsai, Effective Theory of WIMP Dark Matter supplemented by Simplified Models: Singlet-like Majorana fermion case, Physical Review D94 (2016) 065034 [arXiv:1604.02230]. 